Supporting information for:

\title{
Density Functional Calculations of EPR g- and Hyperfine-Coupling Tensors Using the Exact Two-Component (X2C) Transformation and Efficient Approximations to the Two-Electron Spin-Orbit Terms
}

\author{
Artur Wodyński, Martin Kaupp* \\ Technische Universität Berlin, Institut für Chemie, Theoretische Chemie/Quantenchemie, \\ Sekr. C7, Straße des 17. Juni 135, D-10623, Berlin, Germany; \\ email:martin.kaupp@tu-berlin.de.
}

Table S1: Comparison of g-shift and hyperfine tensor components at four-component mDKS and various variants of quasirelativistic two-component DFT levels for a series of $d^{1}$ transition-metal complexes ${ }^{a}$

\begin{tabular}{|c|c|c|c|c|}
\hline & \multicolumn{2}{|c|}{$\Delta g[p p t]$} & \multicolumn{2}{|c|}{$\mathrm{A}[\mathrm{MHz}]$} \\
\hline & $\Delta \mathbf{g}_{\square}$ & $\Delta \mathrm{g}_{\text {| }}$ & $A_{\square}$ & $A_{11}$ \\
\hline \multicolumn{5}{|l|}{$\left[\mathrm{CrOF}_{5}\right]^{2-}$} \\
\hline $4 c-m D K S$ & -61.67 & -50.75 & 52.76 & 132.25 \\
\hline $1 c-X 2 C$ & - & - & 31.76 & 116.87 \\
\hline $2 c-\times 2 c-1 e S O$ & -99.25 & -88.42 & 58.28 & 141.32 \\
\hline 2c-X2C-AMFSO-CG & -51.36 & -41.85 & 52.71 & 127.44 \\
\hline 2c-X2C-AMFSO-C & -58.00 & -47.54 & 53.49 & 129.26 \\
\hline $2 \mathrm{c}-\mathrm{X} 2 \mathrm{C}-\mathrm{mSNSO}$ & -53.51 & -42.47 & 52.84 & 127.33 \\
\hline 2c-X2C-oSNSO & -57.80 & -46.26 & 53.33 & 128.43 \\
\hline 2c-DKH2-AMFSO-C & -57.69 & -46.92 & 53.87 & 129.48 \\
\hline \multicolumn{5}{|l|}{$\left[\mathrm{MoNCl}_{4}\right]^{2-}$} \\
\hline $4 c-m D K S^{a)}$ & -24 & -108 & 161 & 293 \\
\hline $1 c-X 2 C$ & - & - & 157.53 & 256.30 \\
\hline $2 c-X 2 c-1 e S O$ & -34.32 & -150.47 & 162.88 & 304.45 \\
\hline 2c-X2C-AMFSO-CG & -20.75 & -99.62 & 160.61 & 288.50 \\
\hline 2c-X2C-AMFSO-C & -22.03 & -104.27 & 160.85 & 290.08 \\
\hline $2 \mathrm{c}-\mathrm{X} 2 \mathrm{C}-\mathrm{mSNSO}$ & -22.30 & -104.92 & 160.75 & 289.74 \\
\hline 2c-X2C-oSNSO & -23.36 & -108.77 & 160.95 & 291.02 \\
\hline 2c-DKH2-AMFSO-C & -21.80 & -103.61 & 163.50 & 292.62 \\
\hline $\operatorname{Exp}^{S 1}$ & -18 & -96 & - & - \\
\hline \multicolumn{5}{|l|}{$\left[\mathrm{MoOBr}_{5}\right]^{2-}$} \\
\hline $4 c-m D K S^{a)}$ & -67 & 92 & 98 & 200 \\
\hline $1 c-X 2 C$ & - & - & 86.83 & 188.61 \\
\hline $2 c-X 2 C-1 e S O$ & -91.31 & 83.73 & 103.52 & 202.45 \\
\hline 2c-X2C-AMFSO-CG & -61.61 & 92.51 & 98.63 & 192.07 \\
\hline 2c-X2C-AMFSO-C & -64.62 & 92.69 & 99.16 & 192.98 \\
\hline $2 \mathrm{c}-\mathrm{X} 2 \mathrm{C}-\mathrm{mSNSO}$ & -64.93 & 94.37 & 99.10 & 192.20 \\
\hline
\end{tabular}




\begin{tabular}{|c|c|c|c|c|}
\hline & \multicolumn{2}{|c|}{$\Delta \mathrm{g}[\mathrm{ppt}]$} & \multicolumn{2}{|c|}{$\mathrm{A}[\mathrm{MHz}]$} \\
\hline & $\Delta \mathrm{g}_{\square}$ & $\Delta \mathrm{g}_{\mid}$ & $A_{\square}$ & $A_{11}$ \\
\hline $2 c-X 2 C-o S N S O$ & -67.30 & 94.00 & 99.52 & 193.01 \\
\hline 2c-DKH2-AMFSO-C & -63.25 & 93.24 & 100.68 & 194.54 \\
\hline $\operatorname{Exp}^{54}$ & -57 & 87 & 99 & 184 \\
\hline \multicolumn{5}{|l|}{$\left[\mathrm{MoOCl}_{4}\right]^{-}$} \\
\hline $4 c-m D K S^{a)}$ & -58 & -26 & 98 & 223 \\
\hline $1 \mathrm{c}-\mathrm{X} 2 \mathrm{C}$ & - & - & 89.48 & 196.01 \\
\hline $2 c-X 2 c-1 e S O$ & -79.90 & -41.41 & 102.95 & 227.78 \\
\hline 2c-X2C-AMFSO-CG & -53.61 & -22.17 & 98.60 & 216.83 \\
\hline 2c-X2C-AMFSO-C & -56.22 & -23.49 & 99.06 & 217.88 \\
\hline $2 \mathrm{c}-\mathrm{X} 2 \mathrm{C}-\mathrm{mSNSO}$ & -56.65 & -23.80 & 99.02 & 217.44 \\
\hline $2 c-X 2 C-o S N S O$ & -58.72 & -25.07 & 99.39 & 218.32 \\
\hline 2c-DKH2-AMFSO-C & -56.09 & -22.93 & 100.80 & 219.52 \\
\hline $\operatorname{Exp}^{52}$ & -56 & -37 & 145 & 227 \\
\hline \multicolumn{5}{|l|}{$\left[\mathrm{MoOF}_{4}\right]^{-}$} \\
\hline $4 c-m D K S^{a)}$ & -70 & -104 & 126 & 272 \\
\hline $1 \mathrm{c}-\mathrm{X} 2 \mathrm{C}$ & - & - & 116.30 & 234.40 \\
\hline $2 c-X 2 c-1 e S O$ & -95.43 & -142.37 & 132.53 & 278.28 \\
\hline 2c-X2C-AMFSO-CG & -64.20 & -95.88 & 127.23 & 264.30 \\
\hline $2 c-X 2 C-A M F S O-C$ & -67.34 & -99.90 & 127.80 & 265.65 \\
\hline $2 \mathrm{c}-\mathrm{X} 2 \mathrm{C}-\mathrm{mSNSO}$ & -67.91 & -101.22 & 127.77 & 265.33 \\
\hline $2 c-X 2 C-o S N S O$ & -70.36 & -104.70 & 128.22 & 266.46 \\
\hline 2c-DKH2-AMFSO-C & -67.24 & -99.38 & 129.95 & 267.68 \\
\hline $\operatorname{Exp}^{52}$ & -77 & -108 & - & 268 \\
\hline \multicolumn{5}{|l|}{$\left[\mathrm{MoOF}_{5}\right]^{2-}$} \\
\hline $4 c-m D K S^{a)}$ & -109 & -113 & 135 & 278 \\
\hline $1 c-X 2 C$ & - & - & 121.14 & 235.81 \\
\hline $2 c-X 2 c-1 e S O$ & -146.95 & -157.20 & 144.19 & 282.42 \\
\hline 2c-X2C-AMFSO-CG & -100.27 & -103.67 & 137.11 & 267.50 \\
\hline $2 c-X 2 C-A M F S O-C$ & -105.13 & -108.34 & 137.89 & 268.95 \\
\hline $2 \mathrm{c}-\mathrm{X} 2 \mathrm{C}-\mathrm{mSNSO}$ & -105.67 & -109.48 & 137.84 & 268.55 \\
\hline $2 c-X 2 C-o S N S O$ & -109.41 & -113.46 & 138.45 & 269.75 \\
\hline 2c-DKH2-AMFSO-C & -104.81 & -107.76 & 140.04 & 270.99 \\
\hline $\operatorname{Exp}^{\mathrm{S3}}$ & -91 & -128 & 135 & 279 \\
\hline \multicolumn{5}{|l|}{$\left[\mathrm{OsOF}_{5}\right]$} \\
\hline $4 c-m D K S^{a)}$ & -360 & -178 & -448 & -911 \\
\hline $1 \mathrm{c}-\mathrm{X} 2 \mathrm{C}$ & - & - & -377.88 & -631.42 \\
\hline $2 c-X 2 c-1 e s O$ & -438.21 & -230.95 & -479.23 & -889.49 \\
\hline 2c-X2C-AMFSO-CG & -345.29 & -166.73 & -467.99 & -839.27 \\
\hline $2 c-X 2 C-A M F S O-C$ & -353.25 & -171.46 & -469.19 & -843.49 \\
\hline $2 \mathrm{c}-\mathrm{X} 2 \mathrm{C}-\mathrm{mSNSO}$ & -362.61 & -178.69 & -469.80 & -845.68 \\
\hline $2 c-X 2 C-o S N S O$ & -369.79 & -183.21 & -470.77 & -849.69 \\
\hline
\end{tabular}




\begin{tabular}{|c|c|c|c|c|}
\hline & \multicolumn{2}{|c|}{$\Delta g[p p t]$} & \multicolumn{2}{|c|}{$\mathrm{A}[\mathrm{MHz}]$} \\
\hline & $\Delta \mathrm{g}_{\square}$ & $\Delta \mathbf{g}_{\mathrm{II}}$ & $A_{\square}$ & $A_{11}$ \\
\hline 2c-DKH2-AMFSO-C & -353.26 & -170.46 & -481.53 & -855.10 \\
\hline $\operatorname{Exp}^{\mathrm{S12}}$ & -287 & -194 & -480 & -935 \\
\hline \multicolumn{5}{|l|}{$\left[\operatorname{ReNBr}_{4}\right]^{-}$} \\
\hline $4 c-m D K S^{a)}$ & -46 & 82 & -917 & -1915 \\
\hline $1 c-X 2 C$ & - & - & -879.04 & -1520.08 \\
\hline $2 c-X 2 c-1 e S O$ & -67.88 & 79.40 & -910.51 & -1975.57 \\
\hline 2c-X2C-AMFSO-CG & -41.21 & 82.60 & -900.40 & -1859.93 \\
\hline $2 c-X 2 C-A M F S O-C$ & -43.03 & 83.46 & -900.97 & -1868.67 \\
\hline $2 \mathrm{c}-\mathrm{X} 2 \mathrm{C}-\mathrm{mSNSO}$ & -46.35 & 83.97 & -900.02 & -1874.58 \\
\hline $2 c-X 2 C-o S N S O$ & -48.18 & 84.08 & -901.35 & -1883.86 \\
\hline 2c-DKH2-AMFSO-C & -42.57 & 84.99 & -934.08 & -1900.98 \\
\hline $\operatorname{Exp}^{\mathrm{S10}}$ & -29 & 67 & -1013 & -1994 \\
\hline \multicolumn{5}{|l|}{$\left[\mathrm{ReNCl}_{4}\right]^{-}$} \\
\hline $4 c-m D K S^{a)}$ & -79 & -99 & -1081 & -2265 \\
\hline $1 c-X 2 C$ & - & - & -1044.72 & -1689.98 \\
\hline $2 c-X 2 c-1 e s O$ & -104.77 & -121.48 & -1079.26 & -2340.35 \\
\hline 2c-X2C-AMFSO-CG & -73.81 & -92.61 & -1067.30 & -2203.31 \\
\hline $2 c-X 2 C-A M F S O-C$ & -76.11 & -94.38 & -1068.21 & -2214.49 \\
\hline $2 \mathrm{c}-\mathrm{X} 2 \mathrm{C}-\mathrm{mSNSO}$ & -79.99 & -98.44 & -1067.51 & -2223.65 \\
\hline $2 c-X 2 C-o S N S O$ & -82.16 & -100.26 & -1068.99 & -2234.68 \\
\hline 2c-DKH2-AMFSO-C & -75.82 & -93.07 & -1104.57 & -2250.38 \\
\hline $\operatorname{Exp}^{59}$ & -73 & -87 & -1184 & -2263 \\
\hline \multicolumn{5}{|l|}{$\left[\operatorname{ReNF}_{4}\right]^{-}$} \\
\hline 4c-mDKS ${ }^{a)}$ & -121 & -351 & -1587 & -3054 \\
\hline $1 c-X 2 C$ & & & -1527.53 & -2269.38 \\
\hline $2 c-X 2 c-1 e S O$ & -154.99 & -419.59 & -1590.65 & -3151.31 \\
\hline 2c-X2C-AMFSO-CG & -113.90 & -334.80 & -1578.07 & -2970.54 \\
\hline $2 c-X 2 C-A M F S O-C$ & -116.93 & -341.43 & -1579.46 & -2985.62 \\
\hline $2 \mathrm{c}-\mathrm{X} 2 \mathrm{C}-\mathrm{mSNSO}$ & -122.15 & -353.15 & -1578.48 & -2999.77 \\
\hline $2 c-X 2 C-o S N S O$ & -125.06 & -359.08 & -1580.10 & -3014.18 \\
\hline 2c-DKH2-AMFSO-C & -117.10 & -340.41 & -1627.36 & -3034.13 \\
\hline $\operatorname{Exp}^{58}$ & -132 & -353 & -1637 & -3079 \\
\hline \multicolumn{5}{|l|}{$\left[\mathrm{ReOBr}_{4}\right]$} \\
\hline $4 c-m D K S^{a)}$ & -182 & 237 & -626 & -1343 \\
\hline $1 c-X 2 C$ & - & - & -476.67 & -1118.01 \\
\hline $2 c-X 2 c-1 e S O$ & -227.11 & 258.74 & -661.22 & -1333.07 \\
\hline 2c-X2C-AMFSO-CG & -173.14 & 232.19 & -632.07 & -1261.92 \\
\hline $2 c-X 2 C-A M F S O-C$ & -177.44 & 235.78 & -634.70 & -1266.59 \\
\hline 2c-X2C-mSNSO & -183.53 & 240.35 & -636.26 & -1266.29 \\
\hline $2 c-X 2 C-o S N S O$ & -187.39 & 242.67 & -638.91 & -1272.17 \\
\hline 2c-DKH2-AMFSO-C & -177.12 & 237.24 & -656.22 & -1286.41 \\
\hline
\end{tabular}




\begin{tabular}{|c|c|c|c|c|}
\hline & & & & \\
\hline & $\Delta \mathrm{g}_{\square}$ & $\Delta \mathrm{g}_{\mathrm{I}}$ & $A_{\square}$ & $A_{11}$ \\
\hline Exp. $^{\text {S11 }}$ & -232 & 171 & - & - \\
\hline$\left[\operatorname{ReOF}_{5}\right]^{-}$ & & & & \\
\hline $4 c-m D K S^{a)}$ & -362 & -326 & -1372 & -2682 \\
\hline $1 c-X 2 C$ & - & - & -1139.61 & -1877.29 \\
\hline $2 c-X 2 c-1 e S O$ & -434.47 & -404.84 & -1438.50 & -2657.66 \\
\hline 2c-X2C-AMFSO-CG & -346.81 & -308.92 & -1401.02 & -2497.22 \\
\hline $2 c-X 2 C-A M F S O-C$ & -354.41 & -316.39 & -1404.58 & -2510.36 \\
\hline $2 \mathrm{c}-\mathrm{X} 2 \mathrm{C}-\mathrm{mSNSO}$ & -364.01 & -328.59 & -1406.81 & -2520.81 \\
\hline $2 \mathrm{c}-\mathrm{X} 2 \mathrm{C}-\mathrm{OSNSO}$ & -370.56 & -335.39 & -1410.32 & -2533.85 \\
\hline 2c-DKH2-AMFSO-C & -355.20 & -315.45 & -1443.38 & -2545.87 \\
\hline $\operatorname{Exp}^{\mathrm{S12}}$ & -262 & -282 & -1499 & -2878 \\
\hline$\left[\mathrm{TcNBr}_{4}\right]^{-}$ & & & & \\
\hline $4 c-m D K S^{a)}$ & 23 & 171 & -421 & -801 \\
\hline $1 c-X 2 C$ & - & - & -411.86 & -810.52 \\
\hline $2 c-X 2 c-1 e S O$ & 21.61 & 187.72 & -425.21 & -814.10 \\
\hline 2c-X2C-AMFSO-CG & 24.24 & 167.33 & -417.97 & -787.83 \\
\hline 2c-X2C-AMFSO-C & 24.23 & 170.52 & -418.70 & -789.93 \\
\hline $2 \mathrm{c}-\mathrm{X} 2 \mathrm{C}-\mathrm{mSNSO}$ & 24.46 & 172.73 & -417.89 & -786.45 \\
\hline $2 \mathrm{c}-\mathrm{X} 2 \mathrm{C}-\mathrm{oSNSO}$ & 24.29 & 174.68 & -418.59 & -788.48 \\
\hline 2c-DKH2-AMFSO-C & 24.51 & 171.22 & -426.43 & -797.23 \\
\hline $\operatorname{Exp}^{56}$ & 32 & 145 & -360 & -743 \\
\hline$\left[\mathrm{TcNCl}_{4}\right]^{-}$ & & & & \\
\hline $4 c-m D K S^{a)}$ & -5 & 17 & -450 & -930 \\
\hline $1 c-X 2 C$ & - & - & -437.05 & -838.79 \\
\hline $2 c-X 2 c-1 e S O$ & -10.50 & 16.02 & -459.08 & -955.86 \\
\hline 2c-X2C-AMFSO-CG & -4.04 & 17.25 & -450.62 & -916.61 \\
\hline 2c-X2C-AMFSO-C & -4.50 & 17.84 & -451.51 & -920.34 \\
\hline $2 \mathrm{c}-\mathrm{X} 2 \mathrm{C}-\mathrm{mSNSO}$ & -4.64 & 17.93 & -450.85 & -918.13 \\
\hline $2 c-X 2 C-o S N S O$ & -5.12 & 18.10 & -451.61 & -921.30 \\
\hline 2c-DKH2-AMFSO-C & -4.25 & 18.56 & -459.61 & -927.92 \\
\hline $\operatorname{Exp}^{56}$ & -2 & 6 & -402 & -878 \\
\hline$\left[\mathrm{TcNF}_{4}\right]^{-}$ & & & & \\
\hline $4 c-m D K S^{a)}$ & -25 & -91 & -571 & -1153 \\
\hline $1 c-X 2 C$ & - & - & -552.23 & -996.94 \\
\hline $2 c-X 2 c-1 e S O$ & -35.68 & -121.68 & -583.21 & -1190.52 \\
\hline 2c-X2C-AMFSO-CG & -22.19 & -84.62 & -571.88 & -1131.81 \\
\hline 2c-X2C-AMFSO-C & -23.39 & -87.71 & -573.09 & -1137.56 \\
\hline $2 \mathrm{c}-\mathrm{X} 2 \mathrm{C}-\mathrm{mSNSO}$ & -23.68 & -88.61 & -572.44 & -1135.83 \\
\hline $2 c-X 2 C-o S N S O$ & -24.71 & -91.36 & -573.44 & -1140.61 \\
\hline 2c-DKH2-AMFSO-C & -23.14 & -87.03 & -582.97 & -1146.91 \\
\hline $\operatorname{Exp}^{S 5}$ & -12 & -107 & -537 & -1129 \\
\hline
\end{tabular}




\begin{tabular}{|c|c|c|c|c|}
\hline & \multicolumn{2}{|c|}{$\Delta g[p p t]$} & \multicolumn{2}{|c|}{$\mathrm{A}[\mathrm{MHz}]$} \\
\hline & $\Delta \mathrm{g}_{\mathrm{a}}$ & $\Delta \mathbf{g}_{\mid}$ & $A_{\square}$ & $A_{11}$ \\
\hline \multicolumn{5}{|l|}{$\left[\mathrm{WOBr}_{5}\right]^{2-}$} \\
\hline $4 c-m D K S^{a)}$ & -246 & -111 & -141 & -313 \\
\hline $1 c-X 2 C$ & - & - & -115.98 & -216.50 \\
\hline $2 c-X 2 c-1 e S O$ & -303.51 & -153.57 & -147.81 & -318.93 \\
\hline 2c-X2C-AMFSO-CG & -235.27 & -100.55 & -142.45 & -293.35 \\
\hline 2c-X2C-AMFSO-C & -240.89 & -103.95 & -142.93 & -295.34 \\
\hline $2 \mathrm{c}-\mathrm{X} 2 \mathrm{C}-\mathrm{mSNSO}$ & -249.49 & -110.82 & -143.30 & -297.40 \\
\hline $2 c-X 2 C-o S N S O$ & -254.39 & -114.29 & -143.79 & -299.40 \\
\hline 2c-DKH2-AMFSO-C & -240.83 & -102.88 & -147.51 & -299.75 \\
\hline $\operatorname{Exp}^{S 4}$ & -206 & -99 & -105 & - \\
\hline \multicolumn{5}{|l|}{$\left[\mathrm{WOCl}_{4}\right]^{-}$} \\
\hline $4 c-m D K S^{a)}$ & -213 & -200 & -161 & -347 \\
\hline $1 c-X 2 C$ & - & - & -138.34 & -245.57 \\
\hline $2 c-X 2 c-1 e S O$ & -262.23 & -245.74 & -166.55 & -354.67 \\
\hline 2c-X2C-AMFSO-CG & -203.01 & -188.16 & -161.25 & -330.38 \\
\hline $2 c-X 2 C-A M F S O-C$ & -207.92 & -192.39 & -161.71 & -332.35 \\
\hline $2 \mathrm{c}-\mathrm{X} 2 \mathrm{C}-\mathrm{mSNSO}$ & -215.26 & -200.90 & -162.10 & -334.32 \\
\hline $2 c-X 2 C-o S N S O$ & -219.57 & -204.77 & -162.58 & -336.25 \\
\hline 2c-DKH2-AMFSO-C & -207.48 & -190.85 & -166.75 & -337.21 \\
\hline $\operatorname{Exp}^{S 7}$ & -239 & -209 & - & - \\
\hline \multicolumn{5}{|l|}{$\left[\mathrm{WOF}_{5}\right]^{2-}$} \\
\hline $4 c-m D K S^{a)}$ & -354 & -464 & -257 & -473 \\
\hline $1 c-X 2 c$ & - & - & -224.89 & -342.72 \\
\hline $2 c-X 2 c-1 e S O$ & -425.11 & -569.92 & -264.64 & -474.32 \\
\hline 2c-X2C-AMFSO-CG & -339.52 & -440.26 & -259.28 & -444.83 \\
\hline $2 c-X 2 C-A M F S O-C$ & -347.02 & -450.64 & -259.82 & -447.24 \\
\hline $2 \mathrm{c}-\mathrm{X} 2 \mathrm{C}-\mathrm{mSNSO}$ & -356.64 & -468.95 & -260.13 & -449.78 \\
\hline $2 c-X 2 C-o S N S O$ & -363.03 & -478.12 & -260.66 & -452.13 \\
\hline 2c-DKH2-AMFSO-C & -347.18 & -449.91 & -266.78 & -453.99 \\
\hline Exp. ${ }^{\text {S4 }}$ & -330 & -443 & -262 & -469 \\
\hline
\end{tabular}

${ }^{a}$ Computations using the PBE-4OHF hybrid functional, Dyall's TZ basis set for heavy atoms ( $\mathrm{Mn}, \mathrm{Os}, \mathrm{Tc}, \mathrm{Re}, \mathrm{W}, \mathrm{Br}$ ), and IGLO-III basis sets for light atoms $(\mathrm{Cl}, \mathrm{F}, \mathrm{N}, \mathrm{O}$ ). Four component mDKS data obtained with the ReSpect program in Gohr, S.; Hrobárik, P.; Repisky, M.; Komorovsky, S.; Ruud, K.; Kaupp, M. Four-Component Relativistic Density Functional Theory Calculations of EPR g-and Hyperfine-Coupling Tensors Using Hybrid Functionals: Validation on Transition-Metal Complexes with Large Tensor Anisotropies and Higher-Order Spin-Orbit Effects. J. Phys. Chem. A 2015, 119, 12892-12905. Two-component data obtained with the new implementation in the Turbomole program. 
Figure S1: Comparison between results using an exact vs. an RI treatment of the Coulomb operator for small $d^{1}$ complexes $^{a}$

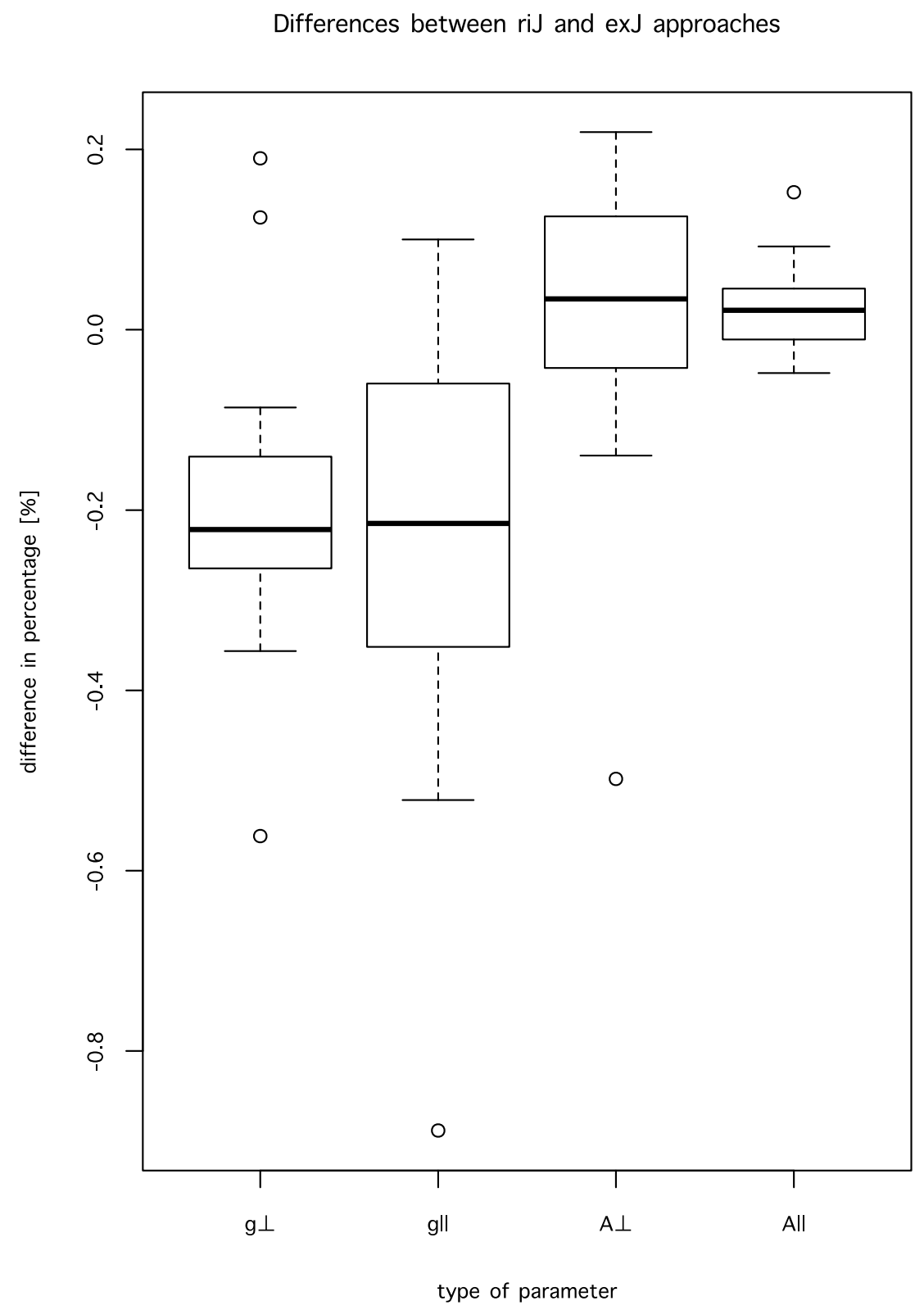

${ }^{\text {a }}$ All calculations performed at 2c-X2C-AMFSO-CG level with Dyall/IGLOIII basis sest and PBE-40HF functional. The RI approximation for the Coulomb operator has been calculated with auxiliary basis sets of Pollak, P.; Weigend, F. Segmented Contracted Error-Consistent Basis Sets of Double- and Triple- $\zeta$ Valence Quality for One-and TwoComponent Relativistic All-Electron Calculations. J. Chem. Theory Comput. 2017, 13, 3696-3705. The exact Coulomb operator has been called in the $2 \mathrm{c}$ module using the \$coulex option. 


\section{References}

S1. Schmitte, J.; Friebel, C.; Weller, F.; Dehnicke, K. Synthese, IR- und EPR-Spektren sowie die Kristallstruktur von (PPh $\left.3{ }_{3} \mathrm{Me}\right)_{2}\left[\mathrm{MoNCl}_{4}\right]$. Z. Anorg. Allg. Chem. 1982, 495, 148-156.

S2. Sunil, K. K.; Rogers, M. T. ESR studies of Some Oxotetrahalo Complexes of Vanadium(IV) and Molybdenum(V). Inorg. Chem. 1981, 20, 3283-3287.

S3. Manoharan, P. T. Ligand Hyperfine Interactions in Molybdenyl and Chromyl Halide Complexes. J. Chem. Phys. 1968, 49, 5510-5519.

S4. van Kemenade, J. T. C., Ligand Hyperfine Interactions in Oxyhalides of Pentavalent Chromium, Molybdenum and Tungsten; Dissertation, Technical University Delft: Delft, Netherland, 1970.

S5. Baldas, J.; Boas, J. F.; Bonnyman, J. Preparation and Properties of Nitridotechnetic(VI) Acid. I. Observation of the ESR Spectrum of the [TcNF4] $]^{-}$Anion in Hydrofluoric Acid Solution. Aust. J. Chem. 1989, 42, 639-648.

S6. Baldas, J.; Boas, J. F.; Bonnyman, J.; Williams, G. A. Studies of Technetium Complexes. The Preparation, Characterisation, and ESR Spectra of Salts of Tetrachloro- and TetrabromoNitridotechnetate(VI): Crystal Structure of Tetraphenylarsonium Tetrachloronitridotechnetate(VI). J. Chem. Soc., Dalton Trans. 1984, 2395-2400.

S7. Kersting, M.; Friebel, C.; Dehnicke, K.; Krestel, M.; Allmann, R. Synthese, IR- und EPRSpektren sowie die Kristallstruktur von HPPh $3\left[\mathrm{WOCl}_{4}(\mathrm{OPPh})\right]$. Z. Anorg. Allg. Chem. 1988, $563,70-78$.

S8 .Voigt, A.; Abram, U.; Kirmse, R. The Existence of [ReNF4] - an EPR Study. Inorg. Chem. Commun. 1998, 1, 141-142.

S9. Voigt, A.; Abram, U.; Böttcher, R.; Richter, U.; Reinhold, J.; Kirmse, R. Q-Band SingleCrystal EPR Study and Molecular Orbital Calculations of $\left[\left(\mathrm{C}_{6} \mathrm{H}_{5}\right)_{4} \mathrm{As}\right]\left[\mathrm{Re}^{\mathrm{VI}} \mathrm{NCl}_{4} / \mathrm{Re}^{\mathrm{V}} \mathrm{OCl}_{4}\right]$. Chem. Phys. 2000, 253, 171-181.

S10. Abram, C.; Braun, M.; Abram, S.; Kirmse, R.; Voigt, A. (NBu4) [ReNCl 4$]$ : Facile Synthesis, Structure, Electron Paramagnetic Resonance Spectroscopy and Reactions. J. Chem. Soc., Dalton Trans. 1998, 231-238.

S11. Borisova, L. V.; Ermakov, A. N.; Plastinina, Y. I.; Prasolova, O. D.; Marov, I. N. Determination of Rhenium Based on the Formation of Rhenium(VI) Oxide Halide Complexes by Spectrophotometry and Electron Spin Resonance Spectroscopy. Analyst 1982, 107, 500504. 
S12. Holloway, J. H.; Hope, E. G.; Raynor, J. B.; Townson, P. T. Magnetic Resonance Studies on Osmium Pentafluoride Oxide. J. Chem. Soc., Dalton Trans. 1992, 1131-1134. 